\title{
DIGIT PATTERNS AND TRANSCENDENTAL NUMBERS
}

\author{
PATRICK MORTON and W. J. MOURANT
}

(Received 1 September 1989; revised 22 December 1989)

Communicated by J. H. Loxton

\begin{abstract}
We use a theorem of Loxton and van der Poorten to prove the transcendence of certain real numbers defined by digit patterns. Among the results we prove are the following. If $k$ is an integer at least $2, P$ is any nonzero pattern of digits base $k$, and $e_{P}^{(r)}(n) \in[0, r-1]$ counts the number of occurrences $(\bmod r)$ of $P$ in the base $k$ representation of $n$, then $\eta\left(e_{P}^{(r)}\right)=\sum_{n=0}^{\infty} e_{P}^{(r)}(n) / r^{n}$ is transcendental except when $k=3, P=1$ and $r=2$. When $(r, k-1)=1$ the linear span of the numbers $\eta\left(e_{P}^{(r)}\right)$ has infinite dimension over $Q$, where $P$ ranges over all patterns base $k$ without leading zeros.
\end{abstract}

1980 Mathematics subject classification (Amer. Math. Soc.) (1985 Revision): 10 F 35, 11 A 63.

\section{Introduction}

This paper is concerned with proving the transcendence of certain real numbers defined using digit patterns. Specifically, let $k$ be an integer at least 2 , and let $P$ be a pattern of digits base $k$. Further let $e_{P}(n)$ denote the number of occurrences of $P$ in the base $k$ representation of $n$, allowing for leading zeros as necessary (see [3], [5], or [13, Section 2]). If $r \geq 2$ is any integer, we define the sequence $e_{P}^{(r)}$ by

$$
e_{P}^{(r)}(n) \equiv e_{P}(n) \quad(\bmod r), \quad \text { where } 0 \leq e_{P}^{(r)}(n) \leq r-1
$$

With these definitions the following results hold.

(C) 1991 Australian Mathematical Society $0263-6115 / 91 \$ A 2.00+0.00$ 
TRANSCENDENCE THeOREM. (a) If $P$ is any nonzero pattern of digits base $k$, the real number

$$
\eta\left(e_{P}^{(r)}\right)=\sum_{n=0}^{\infty} \frac{e_{P}^{(r)}(n)}{r^{n}}
$$

is transcendental, unless $k=3, P=1$ and $r=2$ (in which case $\eta\left(e_{1}^{(2)}\right)=$ $2 / 3$ ).

(b) Let $\mathbb{P}=\{P\}$ be any set of patterns base $k$ with the following properties:

(i) if $P \in \mathbb{P}, P$ has no leading zeros (e.g. $P \neq 0 Q$ for some pattern $Q$ );

(ii) for any pattern $Q$, there is a digit $i$ (base $k$ ) so that $Q i \notin \mathbb{P}$.

If $(r, k-1)=1$, then the corresponding set of real numbers

$$
\left\{\eta\left(e_{P}^{(r)}\right): P \in \mathbb{P}\right\} \cup\{1\}
$$

is linearly independent over $\mathbb{Q}$. Moreover every finite sum

$$
\sum_{P \in \mathbf{P}} c_{P} \eta\left(e_{P}^{(r)}\right), \quad c_{P} \in \mathbb{Q},
$$

is transcendental.

For example, if $P=1^{j}$ is the pattern consisting of the digit 1 repeated $j$ times, the set $\mathbb{P}=\left\{1^{j}: j \geq 1\right\}$ satisfies (i) and (ii) for any $k \geq 2$. Hence the linear span of the numbers $\eta\left(e_{P}^{(r)}\right)$ (for $P \in \mathbb{P}$ ) has infinite dimension over $\mathbb{Q}$ for any $r \geq 2$ for which $(r, k-1)=1$.

Our proof is based on the following theorem of Loxton and van der Poorten (see [8, II, obs. 2.16], [11] and [12]).

TheOREM (Loxton and van der Poorten). Let $r \geq 2$ be fixed and let $\left\{\alpha_{n}\right\}$ be a sequence of integers taken from a finite set. If $\left\{\alpha_{n}\right\}$ is generated by $a$ finite automaton, then $\sum_{n=0}^{\infty} \alpha_{n} / r^{n}$ is either rational or transcendental and is rational if and only if the sequence $\left\{\alpha_{n}\right\}$ is ultimately periodic.

A sequence $\left\{\alpha_{n}\right\}$, whose terms $\alpha_{n}$ are taken from a finite alphabet $A$, is said to be generated by a finite $k$-automaton, or more simply, is $k$-automatic, if the set of distinct sequences of the form

$$
\left\{\alpha_{k^{r} n+j}\right\}, \quad 0 \leq j \leq k^{r}-1,
$$

is finite (see [2, Section 3]). This condition is conveniently expressed in terms of digit substitutions (see [2], [6], [7]). The sequence $\alpha=\left\{\alpha_{n}\right\}$ is said to be a fixed point of a uniform $k$-substitution if there is a rule which replaces each symbol of $A$ by a string of $k$ symbols of $A$, under which $\alpha$ is left fixed. Then $\alpha$ is $k$-automatic if and only if $\alpha$ is the image of a fixed point 
of a $k$-substitution, that is, if and only if $\alpha$ is a sequence derived from a fixed point by renaming or identifying letters of $A$.

We apply the Loxton and van der Poorten theorem to certain groups of sequences that we investigated in [13]. These groups are defined as follows. Let $G$ be an abelian group and $a=\{a(n)\}$ a sequence of elements in $G$. Associated to $a$ are the vectors

$$
X_{n}^{q}=\left(a\left(n k^{q}\right), a\left(n k^{q}+1\right), \ldots, a\left(n k^{q}+k^{q}-1\right)\right),
$$

for each $q \geq 0$, which we refer to as $k^{q}$-segments of $a$. The group $\Gamma_{k}(G)$ is defined to be the set of all sequences for which $a^{-1}(n) X_{n}^{q}$ is periodic, that is, for which there is a positive integer $M$ satisfying

$$
a^{-1}(n) X_{n}^{q}=a^{-1}(m) X_{m}^{q}, \quad \text { for } n \equiv m \quad(\bmod M),
$$

for all $q \geq 0$, where the scalar multiplication is performed by applying $a^{-1}(n)$ to each component of $X_{n}^{q}$ using the operation in $G$. If $G$ is an additive group we write this in the form

$$
X_{n}^{q}-a(n)=X_{m}^{q}-a(m), \quad \text { for } n \equiv m \quad(\bmod M) .
$$

The least positive $M$ for which (4) holds is called the conductor of $a$, and is denoted by $M(a)$. The moduli $M$ for which (4) holds are then exactly the multiples of $M(a)$.

In [13] it is shown that $\Gamma_{k}(G)$ is a group under componentwise multiplication (addition, if $G$ is additive), and that the sequences for which $M(a)$ divides a power of $k$ form a subgroup $\Lambda_{k}(G)$.

An important example is the group $\Gamma_{k}(\mathbb{Z})$. Its subgroup $\Lambda_{k}(\mathbb{Z})$ is generated by the constant sequences and the pattern sequences $e_{P}$, where $P$ is a pattern of digits base $k$ (not all of which are zero), and $e_{P}(n)$ is defined as above. The related sequences $e_{P}^{(r)}$ and $a_{P}$ defined by (1) and

$$
a_{P}(n)=\zeta_{r}^{e_{P}(n)}, \quad \zeta_{r} \text { a primitive } r \text { th root of unity, }
$$

generate the respective groups $\Lambda_{k}\left(\mathbb{Z}_{r}\right)$ (additively) and $\Lambda_{k}\left(\left\langle\zeta_{r}\right\rangle\right)$ (multiplicatively). These are the sequences to which we apply the Loxton and van der Poorten theorem.

We first show (in Section 2) that for finite $G$ the sequences in $\Gamma_{k}(G)$ are all $k$-automatic. This fact allows us to apply the Loxton and van der Poorten theorem to any sequence in $\Lambda_{k}\left(\mathbb{Z}_{r}\right)$ : for a given sequence $e$ in $\Lambda_{k}\left(\mathbb{Z}_{r}\right)$, the number

$$
\eta(e)=\sum_{n=0}^{\infty} \frac{e(n)}{r^{n}}
$$


is rational or transcendental. The sequences $e$ for which $\eta(e)$ is rational are exactly the ultimately periodic sequences in $\Lambda_{k}\left(\mathbb{Z}_{r}\right)$, and these obviously form a subgroup $H_{k}\left(\mathbb{Z}_{r}\right)$ of $\Lambda_{k}\left(\mathbb{Z}_{r}\right)$. A large part of this paper is devoted to determining the subgroup $H_{k}\left(\mathbb{Z}_{r}\right)$. In Section 3 we prove several lemmas concerning the group of periodic sequences in $\Gamma_{k}(G)$, for arbitrary abelian groups $G$.

In Section 4 we determine all periodic sequences in $\Lambda_{k}\left(\left\langle\zeta_{r}\right\rangle\right)$ which have conductor 1 (Theorem 2). They form a cyclic subgroup of $\Lambda_{k}\left(\left\langle\zeta_{r}\right\rangle\right)$ which is generated by the sequence $\left\{\zeta_{r}^{\ell \sigma(n)}\right\}$, where $\sigma(n)$ is the sum of the digits of $n$ base $k$, and $\varepsilon$ is the smallest divisor of $r$ with the property that $r \mid \varepsilon(k-1)$. Using this and a certain endomorphism of $\Lambda_{k}\left(\left\langle\zeta_{r}\right\rangle\right)$ we show that any periodic sequence in $\Lambda_{k}\left(\left\langle\zeta_{r}\right\rangle\right)$ has a period of the form $b M(a)$, where $b \mid(r, k-1)$. If $(r, k-1)=1$ this allows us to give a complete determination of the groups $H_{k}\left(\left\langle\zeta_{r}\right\rangle\right)$ and $H_{k}\left(\mathbb{Z}_{r}\right)$.

The results of Section 4 lead directly to the transcendence results in Section 5 , which are mostly concerned with the transcendence of the numbers in (6) defined using sequences in $\Lambda_{k}\left(\mathbb{Z}_{r}\right)$.

We obtain limited results for the group $\Lambda_{k}\left(\left\langle\zeta_{r}\right\rangle\right)$. We show that when $P$ is an arbitrary nonzero pattern base $k$ with at least 2 digits, and $a_{P}(n)$ is defined in (5), then at least one of the series

$$
\sum_{n=0}^{\infty} \frac{a_{P}^{i}(n)}{r^{n}}, \quad \text { for } 1 \leq i \leq r-1
$$

is transcendental.

Finally, we show that if $a \in \Lambda_{k}\left(\left\langle\zeta_{r}\right\rangle\right)$ with $M(a)=1$, so that $a$ is $k$ multiplicative in Delange's sense [9], then

$$
\sum_{n=0}^{\infty} \frac{a(n)}{r^{n}}
$$

is transcendental if and only if $a$ is not a power of the sequence $\left\{\zeta_{r}^{\varepsilon \sigma(n)}\right\}$ defined above.

We remark that our methods here, as in [13], are elementary. The Loxton and van der Poorten theorem is the only non-elementary component of our argument. We also refer the reader to Allouche [1], which contains results of a related nature, and to Allouche and Shallit [4], which generalizes the notion of an automatic sequence.

We are very grateful to J. P. Allouche and J. Shallit for their remarks concerning an earlier version of this paper. 


\section{Digit substitutions}

Our first theorem shows that the sequences in $\Gamma_{k}(G)$ are $k$-automatic.

TheOREM 1. Let $G$ be a finite abelian group and let a be any sequence in $\Gamma_{k}(G)$. Then for suitable $q$, the sequence $\left\{X_{n}^{q}\right\}$ of $k^{q}$-segments of $a$ is the fixed point of $a k^{r}$-substitution, for some $r \geq 1$. In particular, $a$ is $k$-automatic.

Proof. Let $a \in \Gamma_{k}(G)$ with modulus $M$, let $q \geq 0$ and consider the vector

$$
\left(X_{0}^{q}, X_{1}^{q}, \ldots, X_{M-1}^{q}\right)
$$

For each $q$ we construct a code vector $\kappa_{q}$ of length $M$ which encodes relations among the $X_{n}^{q}$, for $n<M$. We do this as follows: each entry of $\kappa_{q}$ will be an element $(i, g)$ of $\mathbb{Z}_{M} \times G$, where $0 \leq i \leq M-1$ and $g \in G$.

Letting $\kappa_{q}(n)$ denote the $n$th entry of $\kappa_{q}$, we set $\kappa_{q}(0)=(0, \imath)$ where $l$ is the identity in $G$ and $\kappa_{q}(n)=(0, g)$ whenever $X_{n}^{q}=g X_{0}^{q}$. If $m$ is the first index where $X_{m}^{q} \neq g X_{0}^{q}$ for any $g \in G$, set $\kappa_{q}(m)=(1, l)$, and set $\kappa_{q}(n)=(1, g)$ whenever $X_{n}^{q}=g X_{m}^{q}$. Continuing in this manner, we obtain a vector $\kappa_{q}$ for which

$$
\kappa_{q}(n) \in \mathbb{Z}_{M} \times G, \quad \text { for } 0 \leq n \leq M-1
$$

and

$$
\kappa_{q}\left(n_{1}\right)=\kappa_{q}\left(n_{2}\right)(0, g) \quad \text { if and only if } \quad X_{n_{1}}^{q}=g X_{n_{2}}^{q} .
$$

Since there are only finitely many vectors satisfying (7), there must be $q \geq 0$ and $r>0$ for which $\kappa_{q}=\kappa_{q+r}$. For this $q$ and $r$, (8) shows that

$$
X_{n_{1}}^{q}=g X_{n_{2}}^{q} \quad \text { if and only if } \quad X_{n_{1}}^{q+r}=g X_{n_{2}}^{q+r},
$$

for $n_{1}, n_{2}<M$ and $g \in G$. We shall show that the map

$$
X_{n}^{q} \rightarrow X_{n}^{q+r}, \quad n \geq 0,
$$

is equivalent to a digit substitution. First we note that this map is welldefined. Suppose $X_{m}^{q}=X_{n}^{q}$. If $m \equiv m_{1}, n \equiv n_{1}(\bmod M)$ and $m_{1}, n_{1}<$ $M$, then it is an easy exercise using (4), (9) and (4) again (with $q+r$ for $q$ ) to show that $X_{n}^{q+r}=X_{m}^{q+r}$.

Now number the $|G|=m$ elements of $G$ by $g_{0}, g_{1}, \ldots, g_{m-1}$ and replace $g_{i}$ in $X_{n}^{q}$ and $X_{n}^{q+r}$ by $i$. Taken together, the entries in $X_{n}^{q}$ give a digit $d_{n}$ base $m^{k^{q}}$, and the entries in $X_{n}^{q+r}$ give a sequence of $k^{r}$ digits $d_{n, 1}, d_{n, 2}, \ldots, d_{n, k^{r}}$ base $m^{k^{q}}$. The sequence $\left\{d_{n}\right\}$ is therefore fixed by 
the substitution

$$
d_{n} \rightarrow d_{n, 1}, d_{n, 2}, \ldots, d_{n, k^{r}}, \quad \text { for } n \geq 0 .
$$

The concatenated sequences $X_{0}^{q}, X_{1}^{q}, \ldots, X_{n}^{q}, \cdots=X_{0}^{q+r}, X_{1}^{q+r}, \ldots, X_{n}^{q+r}$, ... coincide with the sequence $a$. Since the sequence $a$ is the image of the fixed point $\left\{d_{n}\right\}$ under the map $d_{n} \rightarrow X_{n}^{q}, a$ is $k$-automatic.

COROllary. Let $G=\mathbb{Z}_{m}$ and let $P$ be a nonzero pattern base $k$ of length $d \geq 1$. If $e_{P}^{(m)}(n) \equiv e_{P}(n)(\bmod m)$, where $0 \leq e_{P}^{(m)}(n)<m$, then the sequence $\left\{X_{n}^{d-1}\right\}$ of $k^{d-1}$-segments of the sequence $e_{P}^{(m)}$ is fixed by a $k$-substitution.

Proof. By the proof of the theorem we only need to show for the sequence $e_{P}^{(m)}$ that $X_{n}^{d-1} \rightarrow X_{n}^{d}$ is well-defined, and for this it suffices to prove that

$$
X_{n_{1}}^{d-1} \equiv X_{n_{2}}^{d-1}+\varepsilon \quad(\bmod m) \text { implies } X_{n_{1}}^{d} \equiv X_{n_{2}}^{d}+\varepsilon \quad(\bmod m),
$$

for all $n_{1}$ and $n_{2}$, where $0 \leq \varepsilon \leq m-1$. (We use additive notation since $G$ is an additive group.) Note that (11) holds if and only if

$$
e_{P}^{(m)}\left(n_{1} k^{d-1}+j\right) \equiv e_{P}^{(m)}\left(n_{2} k^{d-1}+j\right)+\varepsilon(\bmod m)
$$

for all $j \in\left[0, k^{d-1}\right)$ implies

$$
e_{P}^{(m)}\left(n_{1} k^{d}+j^{\prime}\right) \equiv e_{P}^{(m)}\left(n_{2} k^{d}+j^{\prime}\right)+\varepsilon \quad(\bmod m)
$$

for all $j^{\prime} \in\left[0, k^{d}\right)$. However the definition of $e_{P}^{(m)}$ implies easily that

$$
e_{P}^{(m)}\left(n_{i} k^{d}+j^{\prime}\right)=e_{P}^{(m)}\left(n_{i} k^{d-1}+j\right)+e_{P}^{(m)}\left(j^{\prime}\right)
$$

where $j^{\prime}=j k+s$ and $0 \leq s \leq k-1$. Thus (12) implies (13).

Alternate proofs of Theorem 1 have been given by Shallit (private communication) and Morton [14].

\section{Properties of periodic sequences in $\Gamma_{k}(G)$}

The lemmas in this section form a basis for the determination in Section 4 of the periodic sequences in $\Lambda_{k}(G)$ for various groups $G$.

Let $G$ be any abelian group. It is easy to show that

LEMMA 1. The set of ultimately periodic sequences in $\Gamma_{k}(G)$ is a subgroup of $\Gamma_{k}(G)$.

LEMMA 2. If $a \in \Gamma_{k}(G)$ is ultimately periodic with period $\rho$, then $a$ is purely periodic with period $\rho$. Every period of $a$ is also a period of the sequence $X^{q}=\left\{X_{n}^{q}\right\}$ of $k^{q}$-segments of $a$. 
Proof. We have

$$
a^{-1}(n) X_{n}^{q}=a^{-1}(m) X_{m}^{q}, \quad \text { for } n \equiv m \quad(\bmod M)
$$

and

$$
a(n+\rho)=a(n), \quad \text { for } n \geq N, \text { for some } N \geq 0 .
$$

It follows easily from (3) that

$$
X_{n+\rho}^{q}=X_{n}^{q} \quad \text { if } n k^{q} \geq N .
$$

Now choose $q$ so that $k^{q} \geq N$. Then for $n, m \geq 1$, the equation

$$
a^{-1}(n+\rho) X_{n+\rho}^{q}=a^{-1}(m+\rho) X_{m+\rho}^{q}, \quad \text { for } n \equiv m \quad(\bmod M)
$$

implies by (16) that

$$
a^{-1}(n+\rho) X_{n}^{q}=a^{-1}(m+\rho) X_{m}^{q}, \quad \text { for } n \equiv m \quad(\bmod M) .
$$

Hence (14) shows that

$$
a(n) a^{-1}(m)=a(n+\rho) a^{-1}(m+\rho)
$$

or

$$
a(m+\rho) a(n)=a(m) a(n+\rho)
$$

for $n \equiv m(\bmod M)$ and $n, m \geq 1$.

Given $m \geq 1$, choose $n \geq N$ so that $n \equiv m(\bmod M)$. Then (15) and (17) imply $a(m)=a(m+\rho)$, if $m \geq 1$. If follows from (15) and (16) that

$$
X_{m}^{q}=X_{m+\rho}^{q}, \quad \text { for } m \geq 1 \text { and all } q \geq 0 \text {. }
$$

Finally, since $M \geq 1$ we see from what we have just shown that

$$
a^{-1}(0) X_{0}^{1}=a^{-1}(M) X_{M}^{1}=a^{-1}(M+\rho) X_{M+\rho}^{1}=a^{-1}(\rho) X_{\rho}^{1} .
$$

Considering the second components in the first and last terms of this equality gives easily that $a(0)=a(\rho)$. This shows that (15) and (16) hold with $N=0$ and proves Lemma 2.

LeMMA 3. If $a \in \Gamma_{k}(G)$ and $a$ is periodic with least period $\rho$, then $\rho=$ $b M(a)$ where $(b, k)=1$.

Proof. The conductor $M(a)$ is the least positive integer $M$ for which (4) holds, that is, for which $n \equiv m(\bmod M)$ implies

(18) $a\left(n k^{q}+j\right) a(m)=a\left(m k^{q}+j\right) a(n), \quad$ for all $j$ with $0 \leq j \leq k^{q}-1$.

This does hold if $M=\rho$, and therefore $M(a) \mid \rho$. 
Now let $M$ be any modulus for $a$ for which $\rho \mid M k^{q}$ for some $q \geq 1$. Taking $j=0$ and $m=n+M$ in (18) gives

$$
a\left(n k^{q}\right) a(n+M)=a\left(n k^{q}+M k^{q}\right) a(n)=a\left(n k^{q}\right) a(n),
$$

which shows that $a(n+M)=a(n)$ for all $n \geq 0$. Therefore $M$ is a period of $a$ and $\rho \mid M$. Hence $\rho \mid M k^{q}$ implies $\rho \mid M$, for any $q \geq 1$. If $\rho=b M(a)$, then a straightforward argument shows that $(b, k)=1$.

LEMMA 4. If $a \in \Gamma_{k}(G)$, then $X^{1}=\left\{X_{n}^{1}\right\} \in \Gamma_{k}\left(G^{k}\right)$, with $M\left(X^{1}\right) \mid M(a)$.

Proof. Define $X_{n}=X_{n}^{1}$ and $\tilde{X}_{n}=\left(X_{n k}, X_{n k+1}, \ldots, X_{n k+k-1}\right)$. Then $X_{n} \in G^{k}$, and $X^{1} \in \Gamma_{k}\left(G^{k}\right)$ if and only if

$$
X_{n}^{-1} \widetilde{X}_{n}=X_{m}^{-1} \widetilde{X}_{m} \quad \text { for } n \equiv m \quad(\bmod \widetilde{M})
$$

for some $\widetilde{M}$. (See [13, Lemma 2].) We show this holds with $\widetilde{M}=M=$ $M(a)$. By definition of $\Gamma_{k}(G)$, the congruence $n \equiv m(\bmod M)$ implies that

$$
X_{n}=a(n) a^{-1}(m) X_{m}
$$

and

$$
X_{n k+j}=a(n k+j) a^{-1}(m k+j) X_{m k+j}, \quad \text { for } 0 \leq j \leq k-1 .
$$

Hence

$$
\begin{aligned}
X_{n}^{-1} \widetilde{X}_{n} & =a^{-1}(n) a(m) X_{m}^{-1}\left(\ldots, a(n k+j) a^{-1}(m k+j) X_{m k+j}, \ldots\right) \\
& =X_{m}^{-1}\left(\ldots, a^{-1}(n) a(m) a(n k+j) a^{-1}(m k+j) X_{m k+j}, \ldots\right) \\
& =X_{m}^{-1}\left(\ldots, X_{m k+j}, \ldots\right)=X_{m}^{-1} \widetilde{X}_{m}, \quad \text { for } n \equiv m(\bmod M),
\end{aligned}
$$

from (20). Since $M\left(X^{1}\right)$ is the least modulus $\widetilde{M}$ for which (19) holds, we have $M\left(X^{1}\right) \mid M(a)$.

Corollary. If $a \in \Gamma_{k}(G)$, then $X^{q}=\left\{X_{n}^{q}\right\} \in \Gamma_{k}\left(G^{k^{q}}\right)$ for $q \geq 1$, and $M\left(X^{q+1}\right) \mid M\left(X^{q}\right)$ for $q \geq 0$. (Note that $X^{0}=a$.)

This follows by induction from Lemma 4.

Let $\rho_{q}=\rho\left(X^{q}\right)$ be the least period of the sequence $X^{q}=\left\{X_{n}^{q}\right\}$, if it exists, for $q \geq 0$. 
Lemma 5. If $a \in \Gamma_{k}(G)$ with least period $\rho=\rho_{0}$ then $X^{q}$ is periodic for all $q \geq 0$ and

(a) $\rho_{q}=\left(\rho_{q}, k\right) \rho_{q+1}$, for $q \geq 0$, and

(b) $\rho=\left(\rho, k^{q}\right) \rho_{q}$.

Proof. To prove $\rho=(\rho, k) \rho_{1}$, note that $\rho_{1}$ is the least integer for which $X_{n+\rho_{1}}^{1}=X_{n}^{1}$ for $n \geq 0$. This holds if and only if

$$
a\left(\left(n+\rho_{1}\right) k+j\right)=a(n k+j), \quad \text { for } 0 \leq j \leq k-1, n \geq 0 .
$$

Equation (21) is satisfied if and only if $\rho_{1} k$ is a period of $a$. Hence $\rho_{1}$ is the least positive integer for which $\rho \mid \rho_{1} k$. By Lemma $2, \rho_{1} \mid \rho$, so $\rho_{1}$ is the least positive integer for which $\left(\rho / \rho_{1}\right) \mid k$, that is, $\rho / \rho_{1}=(\rho, k)$. Applying the same argument to $X^{q}$ and $X^{q+1}$ in place of $a$ and $X^{1}$ proves (a). The proof of (b) is similar.

To summarize, if $a \in \Gamma_{k}(G)$ is a periodic sequence and we apply Lemma 3 to the sequence $X^{q} \in \Gamma_{k}\left(G^{k^{q}}\right)$, we see that

$$
\rho_{q}=b_{q} M_{q}, \quad q \geq 0,
$$

where $\left(b_{q}, k\right)=1$ and $M_{q}=M\left(X^{q}\right)$. (Note that $M_{0}=M(a)$ and $b_{0}=$ $b$, as defined in Lemma 3.) Further, Lemma 5 shows that $\rho_{q+1} \mid \rho_{q}$ and $\left(\rho_{q} / \rho_{q+1}\right) \mid k$. By the Corollary to Lemma 4,

$$
\rho_{q} / \rho_{q+1}=\left(b_{q} / b_{q+1}\right)\left(M_{q} / M_{q+1}\right),
$$

where the first and third quotients lie in $\mathbb{Z}$, and the second involves none of the prime factors of $k$.

Certainly each of the sequences $\rho_{q}, b_{q}$ and $M_{q}$ is constant past a certain point.

LEMMA 6. If $a \in \Gamma_{k}(G)$ is periodic and $M(a)$ divides a power of $k$ (that is, $a \in \Lambda_{k}(G)$ ), then $b=b_{0}=b_{q}$ for $q \geq 0$. If $\rho_{q+1}=\rho_{q}$ then the sequence $X^{q}$ has conductor 1 in $\Gamma_{k}\left(G^{k^{q}}\right)$, and $\left(\rho_{q}, k\right)=1$.

Proof. From (22), $b_{q}=b_{q+1}$, for $q \geq 0$, since $\rho_{q} / \rho_{q+1}$ and $M_{q} / M_{q+1}$ are products of prime factors of $k$ and $\left(b_{q}, k\right)=\left(b_{q+1}, k\right)=1$. Furthermore if $\rho_{q}=\rho_{q+1}$, then by Lemma $5, \rho_{q}$ and $M_{q}$ are relatively prime to $k$. But $M_{q}$ divides $M(a)$, which divides a power of $k$, so $M_{q}=1$. Therefore the sequence $X^{q}$ has conductor 1 in $\Gamma_{k}\left(G^{k^{q}}\right)$ and period $\rho_{q}=b_{q}$ which is prime to $k$. 
We now focus on sequences with conductor 1 . Suppose $a \in \Lambda_{k}(G)$, where $M(a)=1$. Then

$$
X_{n}=a(n) a^{-1}(0) X_{0}, \quad \text { for } n \geq 0 .
$$

Multiplying $a$ by $a^{-1}(0)$ we can assume $a(0)=1$. This gives $X_{n}=a(n) X_{0}$. Writing out both sides of this equation we see that

$$
a(n k+j)=a(n) a(j), \quad \text { for } n \geq 0,0 \leq j \leq k-1 .
$$

Now write $n=j_{t} k^{t}+j_{t-1} k^{t-1}+\cdots+j_{0}$. Then using (23) repeatedly gives

$$
a(n)=a\left(j_{t} k^{t-1}+\cdots+j_{1}\right) a\left(j_{0}\right)=a\left(j_{t}\right) a\left(j_{t-1}\right) \cdots a\left(j_{1}\right) a\left(j_{0}\right) .
$$

We have thus proved the following.

LEMMA 7. If $a \in \Lambda_{k}(G), M(a)=1$, and $a(0)=1$, then $a(n)=$ $\prod_{1=0}^{t} a\left(j_{i}\right)$, where $n=j_{t} j_{t-1} \cdots j_{0}$ (base $k$ ).

The sequences described in Lemma 7 are called $k$-multiplicative sequences by Delange [9]. Combining Lemmas 6 and 7 we see that for every periodic sequence $a \in \Lambda_{k}(G)$ there is a $q$ for which $\left(X_{0}^{q}\right)^{-1} X^{q} \in \Gamma_{k}\left(G^{k^{q}}\right)$ is $k$ multiplicative.

\section{Periodicity results}

We first determine the periodicity of sequences defined by patterns consisting of single digits. We use this in Theorem 3 to describe the possible periods of periodic sequences in $\Lambda_{k}(\langle\zeta\rangle)$, where $\zeta$ is an $r$ th root of unity.

THEOREM 2. Let $i$ be a digit base $k$ and $a_{i}(n)=\zeta^{e_{i}(n)}$, where $\zeta=\zeta_{r}$ is an rth root of unity. If $S$ is a non-empty set of nonzero digits base $k$, and

$$
a_{s}=\prod_{i \in S} a_{i}^{\varepsilon_{i}}
$$

where $r$ does not divide $\varepsilon_{i}$, then $a_{s}$ is not ultimately periodic, unless $a_{s}(n)=$ $\zeta^{e_{1} \sigma(n)}$ where $\sigma=e_{1}+2 e_{2}+\cdots+(k-1) e_{k-1}$, and $r /\left(r, \varepsilon_{1}\right) \mid(k-1)$. In this case $a_{s}$ has least period $r /\left(r, \varepsilon_{1}\right)$, and $S=\left\{i \mid i \neq 0\left(\bmod r /\left(r, \varepsilon_{1}\right)\right)\right\}$.

Proof. Assume $a_{s}$ is ultimately periodic and that $\rho$ is the least period of $a_{s}$. We know $M\left(a_{s}\right)=1$ in $\Gamma_{k}(\langle\zeta\rangle)$ because the patterns in $S$ all have length 
1. Lemmas 2 and 3 show that $a_{s}$ is purely periodic and that $(\rho, k)=1$. Hence

$$
a_{s}(n+m \rho)=a_{s}(n), \quad \text { for } m, n \geq 0 .
$$

I. Assume $m \rho$ has $j$ as its last digit. If $i$ is any digit for which $i+j<k$, then by Lemma 7 ,

$$
a_{s}(i+m \rho)=a_{s}(m \rho) a_{s}^{-1}(j) a_{s}(i+j)=a_{s}^{-1}(j) a_{s}(i+j) .
$$

From (25) with $n=i$ it follows that

$$
a_{s}(i+j)=a_{s}(i) a_{s}(j) .
$$

In fact this holds for any $i, j \in[0, k-1]$ for which $i+j<k$. To see this we need only check that any $j$ is the last digit of $m \rho$ for suitable $m$. This holds whenever $m \rho \equiv j(\bmod k)$ and since $k$ and $\rho$ are relatively prime, this congruence has a solution $m$ for any $j \in[0, k-1]$.

II. Assume $m \rho$ has $0 j$ as its last two digits. If $i$ is any digit for which $i+j \geq k$, Lemma 7 gives

$$
\begin{aligned}
a_{s}(i+m \rho) & =a_{s}(m \rho) a_{s}^{-1}(j) a_{s}(1) a_{s}(i+j-k) \\
& =a_{s}^{-1}(j) a_{s}(i+j-k) a_{s}(1) .
\end{aligned}
$$

From (25) with $n=i$ we see that

$$
a_{s}(i+j-k)=a_{s}(i) a_{s}(j) a_{s}^{-1}(1) .
$$

Again, (27) holds for any $j$, since the congruence $m \rho \equiv 0 \cdot k+j\left(\bmod k^{2}\right)$ is always solvable.

We now define $\alpha: \mathbb{Z}_{k} \rightarrow\langle\zeta\rangle$ by $\alpha(i)=a_{s}(i), 0 \leq i \leq k-1$. There are two cases.

Case A: $1 \notin S$. By (24), $a_{s}(1)=1$. Here (26) and (27) imply that $\alpha$ is a homomorphism. But $\alpha(1)=1$, so the kernel of $\alpha$ contains all of $\mathbb{Z}_{k}$. Hence $a_{s}(i)=1$ for all $i \in[0, k-1], S$ is empty by (24), contradicting our assumption.

Case B: $1 \in S$. Here $a_{s}(1)=\zeta^{\ell_{1}} \neq 1$. Now (26) implies by induction that $a_{s}(j)=\zeta^{j \varepsilon_{1}}$ for $j \in[0, k-1]$. For general $n=j_{t} j_{t-1} \cdots j_{0}$ (base $k$ ), Lemma 7 gives

$$
\begin{aligned}
a_{s}(n) & =a_{s}\left(j_{t}\right) a_{s}\left(j_{t-1}\right) \cdots a_{s}\left(j_{0}\right) \\
& =\zeta^{\left(j_{t}+j_{t-1}+\cdots+j_{0}\right) \varepsilon_{1}}=\zeta^{\sigma(n) \varepsilon_{1}},
\end{aligned}
$$

where $\sigma(n)=j_{t}+j_{t-1}+\cdots+j_{0}=e_{1}(n)+2 e_{2}(n)+\cdots+(k-1) e_{k-1}(n)$. By assumption, $\sigma(n) \varepsilon_{1}$ has least period $\rho(\bmod r)$. With $g=\left(r, \varepsilon_{1}\right)$, this says 
$\sigma(n)$ has least period $\rho\left(\bmod r^{\prime}\right)$, where $r^{\prime}=r / g$. We write $a_{s}(n)=\zeta_{1}^{\sigma(n)}$, where $\zeta_{1}=\zeta^{\varepsilon_{1}}$ is a primitive $r^{\prime}$ th root of unity. Using (27), we have

$$
\begin{aligned}
1 & =a_{s}(0)=a_{s}(k-1+1-k) \\
& =a_{s}(k-1) a_{s}(1) a_{s}^{-1}(1)=\zeta^{(k-1) \varepsilon_{1}}=\zeta_{1}^{k-1}
\end{aligned}
$$

which implies that $r^{\prime}=r /\left(r, \varepsilon_{1}\right)$ divides $k-1$.

From the fact that $\sigma(n) \equiv n\left(\bmod r^{\prime}\right)$, if $r^{\prime}$ divides $k-1$, it follows that the least period of $\sigma(n)\left(\bmod r^{\prime}\right)$ is $r^{\prime}$ and hence $\rho=r^{\prime}=r /\left(r, \varepsilon_{1}\right)$. The characterization of the set $S$ follows from the formula

$$
a_{s}(n)=\zeta^{\varepsilon_{1} \sigma(n)}=\prod_{i=1}^{k-1} a_{i}^{e_{1} i} .
$$

Corollary 1. The only $k, i$, and $r \geq 2$ for which $a_{i}=\zeta^{e_{i}}$ can be periodic are $k=3, i=1$, and $r=2$. In this case $e_{1}$ (base 3 ) is periodic $(\bmod 2)$ with period 2.

Proof. If $a_{i}=\zeta^{e_{i}}$ is periodic, then Theorem 2 implies that $i=1$ (so that $\varepsilon_{1} \neq 0$ ) and

$$
e_{1}(n) \equiv \sigma(n) \quad(\bmod r), \quad \text { for } n \geq 0 .
$$

Thus we have

$$
2 e_{2}(n)+\cdots+(k-1) e_{k-1}(n) \equiv 0 \quad(\bmod r), \quad \text { for } n \geq 0 .
$$

Note that $k \geq 3$, since $k=2$ would give $r=1$. Setting $n=2$ in (28) gives $2 \equiv 0(\bmod r)$, and so $r=2$. It now follows easily that $k=3$, for if $k \geq 4$, putting $n=3$ in $(28)$ gives $3 \equiv 0(\bmod 2)$.

The following additive form of Theorem 2 will be useful.

COROLlaRY 2. Let $S$ be a non-empty set of nonzero digits base $k$, and

$$
e_{s} \equiv \sum_{i \in S} \varepsilon_{i} e_{i} \quad(\bmod r), \quad \text { for } n \geq 0,
$$

where $\varepsilon_{i} \not \equiv 0(\bmod r)$. Then the sequence $e_{s}$ is not ultimately periodic $(\bmod r)$ unless $e_{s}(n) \equiv \varepsilon_{1} \sigma(n)(\bmod r)$ and $r /\left(r, \varepsilon_{1}\right) \mid(k-1)$.

Corollary 3. If $S$ is a non-empty set of nonzero digits base $k$, and $\varepsilon_{i}$ is a nonzero integer for $i \in S$, then the integer sequence

$$
e_{s}=\sum_{i \in S} \varepsilon_{i} e_{i}
$$

is not ultimately periodic. 
Proof. Let $r$ be a prime larger than $\max \left(k, \varepsilon_{1}, \ldots, \varepsilon_{k-1}\right)$. If $e_{s}$ is ultimately periodic, then $e_{s}$ is ultimately periodic $(\bmod r)$. This is impossible by Corollary 2 , since $r>k-1$.

THEOREM 3. Let $\zeta$ be an rth root of unity. If $a \in \Lambda_{k}(\langle\zeta\rangle)$ is ultimately periodic, then $a$ is purely periodic with period equal to $b M(a)$, where $b \mid(r, k-1)$. If $(r, k-1)=1$, then a has period $M(a)$.

Proof. Assume $a \in \Lambda_{k}(\langle\zeta\rangle)$ is ultimately periodic, and $a(0)=1$. Then $a$ is purely periodic, and for some $q, X^{q}=\left\{X_{n}^{q}\right\} \in \Lambda_{k}\left(\langle\zeta)^{k^{q}}\right)$ is periodic with period $\rho_{q}$, where $\left(\rho_{q}, k\right)=1$, and $M_{q}=M\left(X^{q}\right)=1$, by Lemma 6 .

Set

$$
Y_{n}=\left(X_{0}^{q}\right)^{-1} X_{n}^{q}
$$

Then $M\left(\left\{Y_{n}\right\}\right)=1, Y_{0}$ is the identity in $G=\langle\zeta\rangle^{k^{q}}$, and $\left\{Y_{n}\right\}$ has least period $\rho_{q}$.

Now let $\varphi_{i}: G \rightarrow\langle\zeta\rangle$ be the projection homomorphism of $G$ which takes a vector to its $i$ th coordinate, and define the sequence $\alpha_{i}$ by

$$
\alpha_{i}(n)=\varphi_{i}\left(Y_{n}\right) .
$$

Since $\varphi_{i}: G \rightarrow\langle\zeta\rangle, \alpha_{i} \in \Gamma_{k}(\langle\zeta\rangle)$ by results of [13] (equations (34) and $\left.\left(34^{\prime}\right)\right)$. It is easily checked that $M\left(\alpha_{i}\right)=1$ and that $\alpha_{i}$ is periodic with $\rho\left(\alpha_{i}\right) \mid \rho_{q}$.

By results of [13] (see Theorem 7), $M\left(\alpha_{i}\right)=1$ implies that $\alpha_{i}$ is a product of pattern sequences $a_{j}$, where $j$ runs over a set $S$ of digits base $k$ :

$$
\alpha_{i}=\prod_{j \in S} a_{j}^{\varepsilon_{j}} .
$$

Since $\alpha_{i}$ is periodic, Theorem 2 now gives that $\rho\left(\alpha_{i}\right) \mid(r, k-1)$. But since

$$
Y_{n}=\left(\alpha_{1}(n), \alpha_{2}(n), \ldots, \alpha_{k^{q}}(n)\right),
$$

we have $\rho_{q}=1$. c. m. ${ }_{i} \rho\left(\alpha_{i}\right)$, and therefore $\rho_{q} \mid(r, k-1)$. It follows that $\rho_{q}=b_{q}=b$, by Lemma 6 , and so Lemma 3 gives that $\rho(a)=b M(a)$, where $b \mid(r, k-1)$.

REMARK. Exactly the same proof shows that any ultimately periodic sequence in $\Lambda_{k}(\mathbb{Z})$ is purely periodic with period equal to $M(a)$, a power of $k$, and that a periodic sequence $a$ in $\Lambda_{k}\left(\mathbb{Z}_{r}\right)$ has a least period of the form $b M(a)$, where $b \mid(r, k-1)$. 
In [13, Theorem 8] it was proved that

$$
e_{P}=e_{0 P}+e_{1 P}+\cdots+e_{(k-1) P},
$$

where $P$ is a pattern base $k$ and $i P$ is the pattern consisting of the digit $i$ followed by $P$. The following theorem complements this result and generalizes [13, Theorem 10]. We recall from [13] the notation $v(P)$, which denotes the value of $P$ considered as an integer base $k$.

TheOREM 4. If $P$ is a nonzero pattern base $k$ of length $d$ and $P i$ is the pattern consisting of the pattern $P$ followed by the digit $i$, then the sequence

$$
h_{P}=e_{P}-e_{P 0}-e_{P 1}-\cdots-e_{P(k-1)}
$$

in $\Lambda_{k}(\mathbb{Z})$ is periodic with period $k^{d}$ and we have

$$
h_{P}(n)= \begin{cases}0 & \text { if } n \not \equiv v(P)\left(\bmod k^{d}\right), \\ 1 & \text { if } n \equiv v(P)\left(\bmod k^{d}\right)\end{cases}
$$

The sequences $h_{P}$ together with the constant sequences generate $H_{k}(\mathbb{Z})$, the subgroup of periodic sequences in $\Lambda_{k}(\mathbb{Z})$. If $(r, k-1)=1$, the sequences

$$
h_{P}^{(r)}=e_{P}^{(r)}-e_{P 0}^{(r)}-e_{P 1}^{(r)}-\cdots-e_{P(k-1)}^{(r)}
$$

generate $H_{k}\left(\mathbb{Z}_{r}\right)$.

Proof. Equation (31) follows from the fact that the occurrences of $P$ in the $k$-adic expression for $n$ coincide with the occurrences of $P i$ for $0 \leq i \leq k-1$, with the possible exception of an occurrence of $P$ at the end. The latter can occur if and only if $n \equiv v(P)\left(\bmod k^{d}\right)$.

Now let $a$ be any sequence in $H_{k}(\mathbb{Z})$. By the remark following Theorem 3, $a$ is purely periodic, with period equal to $M(a)=k^{d}$ for some $d$. It then follows easily from (31) that

$$
a=a(0)+\sum(a(v(P))-a(0)) h_{P},
$$

where $P$ runs over all nonzero patterns base $k$ of length $d$. Exactly the same argument works for the group $H_{k}\left(\mathbb{Z}_{r}\right)$.

REMARK. If $P=0^{*}$, the pattern consisting of $d 0$ 's, then in place of (31) we have

$$
h_{0^{*}}(n)= \begin{cases}0 & \text { if } n \equiv 0\left(\bmod k^{d}\right), \\ -1 & \text { if } n \neq 0\left(\bmod k^{d}\right) .\end{cases}
$$

Let $\zeta \neq 1$ be any $r$ th root of unity for which $(r, k-1)=1$ and let $a_{P}(n)=\zeta^{e_{P}(n)}$. We define

$$
b_{P}(n)=a_{P}(n) a_{P 0}^{-1}(n) a_{P 1}^{-1}(n) \cdots a_{P(k-1)}^{-1}(n) .
$$


By (31)

$$
b_{P}(n)= \begin{cases}1 & \text { if } n \not \equiv v(P)\left(\bmod k^{d}\right), \\ \zeta & \text { if } n \equiv v(P)\left(\bmod k^{d}\right)\end{cases}
$$

COROLLARY TO THEOREM 4. If $(r, k-1)=1$ the sequences $b_{p}$, together with the constant sequences in $\Gamma_{k}(\langle\zeta\rangle)$, generate the subgroup $H_{k}(\langle\zeta\rangle)$ of periodic sequences in $\Lambda_{k}(\langle\zeta\rangle)$.

The proof is analogous to the proof of Theorem 4.

The next theorem extends Corollary 1 of Theorem 2 to patterns of length greater than 1 .

THEOREM 5. Let $P$ be a nonzero pattern of digits base $k$ having length $d>1$. If $\zeta$ is an rth root of unity then neither $e_{P}^{(r)}$ nor $a_{P}=(\zeta)^{e_{P}}$ is periodic.

Proof. We show that $e_{P}$ is not periodic $(\bmod r)$, where $P$ has $d$ digits. If $e_{P}$ is periodic $(\bmod r)$, Theorem 3 implies that $e_{P}^{(r)}$ is purely periodic with period $b M\left(e_{P}^{(r)}\right)$, where $b \mid k-1$. From [13] we know that $M\left(e_{P}^{(r)}\right) \mid k^{d-1}$. Therefore Lemma 5(b) implies that the period $\rho_{d-1}$ of the sequence $X^{d-1}=$ $\left\{X_{n}^{d-1}\right\}$ is $b$, and hence that $k-1$ is a period of $X^{d-1}$. But since

$$
X_{n}^{d-1}=\left(e_{P}^{(r)}\left(n k^{d-1}\right), e_{P}^{(r)}\left(n k^{d-1}+1\right), \ldots, e_{P}^{(r)}\left(n k^{d-1}+k^{d-1}-1\right)\right),
$$

we have that

$$
\begin{aligned}
& e_{P}^{(r)}\left(n k^{d-1}+j\right)=e_{P}^{(r)}\left((n+k-1) k^{d-1}+j\right) \\
& \qquad \text { for } n \geq 0 \text { and } 0 \leq j \leq k^{d-1}-1 .
\end{aligned}
$$

Case 1. If the base $k$ representation of $P$ is $0 p_{2} p_{3} \cdots p_{d}$, choose $n$ and $j$ with base $k$ representations

$$
n=11 \cdots 10 \text {, with } d+1 \text { digits, and } j=p_{2} p_{3} \cdots p_{d} .
$$

Then the base $k$ representations of $n k^{d-1}+j$ and $(n+k-1) k^{d-1}+j$ are, respectively,

$$
11 \cdots 10 p_{2} p_{3} \cdots p_{d} \text { and } 11 \cdots 1(k-1) p_{2} p_{3} \cdots p_{d} .
$$

Thus

$$
e_{P}\left(n k^{d-1}+j\right)=e_{P}\left((n+k-1) k^{d-1}+j\right)+1 .
$$

Hence (33) cannot hold. 
Case 2. If the base $k$ representation of $P$ is $p_{1} p_{2} p_{3} \cdots p_{d}$, with $p_{1} \geq 1$, choose $n$ and $j$ with base $k$ representations

$$
n=10 \cdots 00 p_{1} \text {, with } d+2 \text { digits, and } j=p_{2} p_{3} \cdots p_{d} \text {. }
$$

Then the base $k$ representations of $n k^{d-1}+j$ and $(n+k-1) k^{d-1}+j$ are, respectively,

$$
10 \cdots 00 p_{1} p_{2} p_{3} \cdots p_{d} \text { and } 10 \cdots 01\left(p_{1}-1\right) p_{2} p_{3} \cdots p_{d} \text {. }
$$

The same conclusion holds as in Case 1 unless $1\left(p_{1}-1\right) p_{2} p_{3} \cdots p_{d-1}=P$. In this case replace $j$ by $j^{\prime}=j+1$ if $p_{d}=0$ or by $j^{\prime}=j-1$ if $p_{d} \geq 1$. Then clearly

$$
e_{P}\left(n k^{d-1}+j^{\prime}\right)=e_{P}\left((n+k-1) k^{d-1}+j^{\prime}\right)-1 .
$$

Hence (33) cannot hold in this case either, and $e_{P}$ cannot be periodic $(\bmod r)$.

LEMMA 8. If $P$ is any pattern base $k$. Then

$$
h_{0 P}=h_{P}-h_{1 P}-h_{2 P}-\cdots-h_{(k-1) P} .
$$

The same relationship exists between $h_{i P}^{(r)}$ and $h_{P}^{(r)}$.

This is immediate from (30) and the definition of $h_{P}$ (see Theorem 4).

LeMma 9. The sequences $e_{P}^{(r)}$, for patterns $P$ (base $k$ ) with no leading zeros, are independent in $\Lambda_{k}\left(\mathbb{Z}_{r}\right)$.

Proof. We have to show that

$$
\sum_{P} c_{P} e_{P}^{(r)}=0 \text { in } \Lambda_{k}\left(\mathbb{Z}_{r}\right), \quad c_{P} \in \mathbb{Z}_{r},
$$

implies all $c_{P} \equiv 0(\bmod r)$. But this follows easily from the fact that $e_{P}(n)=$ 1 if $n=v(P)$ and $e_{P}(n)=0$ if $n<v(P)$. (See also the argument in the proof of Theorem 9 in [13].)

Definition. Fix $k$ and let $\mathbb{P}=\{P\}$ be a set of nonzero patterns base $k$ with no leading zeros. Then $\mathbb{P}$ is called a a set of independence if it has the property that for all patterns $P$ base $k$ there exists a digit $i$ (base $k$ ) so that $P i \notin \mathbb{P}$.

TheOREM 6. If $(r, k-1)=1$ and $\mathbb{P}$ is a set of independence then $\left\{e_{P}^{(r)} ; P \in \mathbb{P}\right\}$ is a set of independent elements in the quotient group

$$
\Lambda_{k}\left(\mathbb{Z}_{r}\right) / H_{k}\left(\mathbb{Z}_{r}\right) \text {. }
$$


Proof. We have to show that

$$
\sum_{P \in \mathbb{P}} c_{P} e_{P}^{(r)} \in H_{k}\left(\mathbb{Z}_{r}\right), \quad c_{P} \in \mathbb{Z}_{r},
$$

implies $c_{P} \equiv 0(\bmod r)$ for all $P \in \mathbb{P}$. Assume (34) holds. Then by Theorem 4 and Lemma 8 we can write

$$
\sum_{P \in \mathbf{P}} c_{P} e_{P}^{(r)}=\sum_{Q} d_{Q} h_{Q}^{(r)}
$$

where the sum is over all patterns $Q$ base $k$ with no leading zeros, and only a finite number of $d_{Q}$ are nonzero $(\bmod r)$. Rewriting the right side of (35) gives

$$
\sum_{P \in \mathbf{P}} c_{P} e_{P}^{(r)}=\sum_{Q} d_{Q}\left(e_{Q}^{(r)}-e_{Q 0}^{(r)}-\cdots-e_{Q(k-1)}^{(r)}\right)=\sum_{Q}\left(d_{Q}-d_{Q^{\prime}}\right) e_{Q}^{(r)},
$$

where $Q=Q^{\prime} i$ for some $i$. Suppose some $d_{Q} \not \equiv 0(\bmod r)$. By assumption on $\mathbb{P}$ there is a digit $j$ base $k$ such that $Q j \notin \mathbb{P}$. Moreover there exist digits $j_{1}(=j), j_{2}, \cdots, j_{n}$, for all $n \geq 0$, such that $Q_{n}=Q j_{1} j_{2} \cdots j_{n} \notin \mathbb{P}$. Thus Lemma 9 gives that $d_{Q_{n}}-d_{Q_{n-1}} \equiv 0(\bmod r)$, for all $n \geq 1$. Therefore $d_{Q_{n}} \equiv d_{Q_{n-1}} \equiv \cdots \equiv d_{Q} \not \equiv 0(\bmod r)$ for all $n$, and so there are infinitely many nonzero $d_{Q}$ 's. This contradiction shows that all $d_{Q} \equiv 0(\bmod r)$ in (35), which implies all $c_{P} \equiv 0(\bmod r)$.

\section{Transcendence results}

Theorems 7 and 8 in this section are immediate consequences of Theorem 1 , the results of Section 4, and the Loxton and van der Poorten theorem. Theorem 7 is concerned with sequences defined by single digit patterns, while Theorem 8 gives a necessary and sufficient condition for the number $\eta(e)$ in (6) to be transcendental, where $e$ is a sequence in $\Lambda_{k}\left(\mathbb{Z}_{r}\right)$ with $(r, k-1)=1$. From Corollary 2 to Theorem 2 we have

THEOREM 7. Let $S$ be a non-empty set of nonzero digits base $k$, and

$$
e_{s}(n) \equiv \sum_{i \in S} \varepsilon_{i} e_{i}(n) \quad(\bmod r),
$$

where $e_{s}(n) \in[0, r-1]$ and $\varepsilon_{i} \not \equiv 0(\bmod r)$. If either

(A) $(r, k-1)>1$ and $e_{s}(n) \not \equiv \varepsilon \sigma(n)(\bmod r)$ for any integer $\varepsilon \not \equiv 0$ $(\bmod r)($ with $\sigma$ as in Theorem 2), or

(B) $(r, k-1)=1$, then

is a transcendental number.

$$
\sum_{n=0}^{\infty} \frac{e_{s}(n)}{r^{n}}
$$


REMARK. If $1 \notin S$, then condition (A) holds.

Corollary. If $e_{i}^{(r)}(n)$ is defined as in (1) with $P=i$, a digit base $k$, then unless $(k, i, r)=(3,1,2)$ the number

$$
\eta\left(e_{i}^{(r)}\right)=\sum_{n=0}^{\infty} \frac{e_{i}^{(r)}(n)}{r^{n}}
$$

is transcendental. If $(k, i, r)=(3,1,2)$, then

$$
\eta\left(e_{1}^{(2)}\right)=\sum_{n=0}^{\infty} \frac{e_{1}^{(2)}(n)}{2^{n}}=\sum_{n=0}^{\infty} \frac{1}{2^{2 n+1}}=\frac{2}{3} .
$$

THEOREM 8. If $e \in \Lambda_{k}\left(\mathbb{Z}_{r}\right)$ where $e(n) \in[0, r-1]$, then the number $\eta(e)$ defined as in (6) is transcendental if $e \notin H_{k}\left(\mathbb{Z}_{r}\right)$ and rational if $e \in H_{k}\left(\mathbb{Z}_{r}\right)$. If $(r, k-1)=1$ then $e \in H_{k}\left(\mathbb{Z}_{r}\right)$ if and only if $e$ can be expressed as

$$
e \equiv e(0)+\sum_{Q} c_{Q} h_{Q}(\bmod r),
$$

where $h_{Q}=e_{Q}-e_{Q 0}-e_{Q 1}-\cdots-e_{Q(k-1)}$, for patterns $Q$ base $k$, all having the same length.

COROllary. If $P$ is any nonzero pattern of digits base $k$ having length at least 2 , and if $e_{P}^{(r)}(n)$ is defined as in (1), then the number

$$
\eta\left(e_{P}^{(r)}\right)=\sum_{n=0}^{\infty} \frac{e_{P}^{(r)}(n)}{r^{n}}
$$

is transcendental.

This corollary follows immediately from Theorem 5 .

THEOREM 9. If $\mathbb{P}$ is a set of independence for the base $k$, and $(r, k-1)=$ 1 , then the set

$$
\left\{\eta\left(e_{P}^{(r)}\right): P \in \mathbb{P}\right\} \cup\{1\}
$$

is linearly independent over $\mathbb{Q}$. Moreover every finite sum

$$
\sum_{P \in \mathbb{P}} c_{P} \eta\left(e_{P}^{(r)}\right), \quad c_{P} \in \mathbb{Q},
$$

is transcendental. 
Proof. Assume there is a dependence among 1 and the $\eta\left(e_{P}^{(r)}\right)$ :

$$
\sum_{P \in \mathbb{P}} c_{P} \eta\left(e_{P}^{(r)}\right)=c
$$

where, without loss of generality, $c_{P} \in \mathbb{Z}$ for $P \in \mathbb{P}$, not all $c_{P} \equiv 0(\bmod r)$, and $c \in \mathbb{Q}$. Rewriting (38) gives

$$
\sum_{n=0}^{\infty} \frac{\sum_{P \in \mathbb{P}} c_{P} e_{P}^{(r)}(n)}{r^{n}}=c .
$$

By Theorem 1, each of the sequences $e_{P}^{(r)}$ is $k$-automatic, and so the sequence

$$
a=\sum_{P \in \mathbb{P}} c_{P} e_{P}^{(r)}
$$

is also $k$-automatic. Note that the values $a(n)$ do not necessarily lie in $[0, r-1]$, but that they are from a finite set. By Theorem $6, a$ is not periodic $(\bmod r)$ and therefore is not a periodic sequence. The Loxton and van der Poorten theorem now shows that (39) is impossible. The transcendence statement follows in the same way.

We now prove the following multiplicative result.

THEOREM 10. Let $P$ be an arbitrary nonzero pattern base $k$ with at least 2 digits, and let $a_{P}(n)=(\zeta)^{e_{P}(n)}$, as before, where $\zeta$ is a primitive rth root of unity. Then at least one of the series

$$
\sum_{n=0}^{\infty} \frac{a_{p}^{i}(n)}{r^{n}}, \quad \text { for } 1 \leq i \leq r-1,
$$

is transcendental.

Proof. The key to the proof lies in relating (40) to the number

$$
\sum_{n=0}^{\infty} \frac{e_{P}^{(r)}(n)}{r^{n}}
$$

and appealing to the corollary to Theorem 8. In general, suppose $a(n)=$ $\zeta^{e(n)}$, where $e(n) \in\{0,1, \ldots, r-1\}$. Then

$$
e(n)=\sum_{i=0}^{r-1} c_{i} a^{i}(n)
$$

for suitable constants $c_{i} \in \mathbb{Q}(\zeta)$ (independent of the particular sequence $e(n))$, as is easily seen by replacing $e(n)$ by its $r$ possible values $0, \ldots$, $r-1$ and solving the resulting linear system, whose determinant is a nonzero 
Vandermonde determinant. Hence

$$
\sum_{n=0}^{\infty} \frac{e_{P}^{(r)}(n)}{r^{n}}=c_{0} \frac{r}{r-1}+\sum_{i=1}^{r-1} c_{i} \sum_{n=0}^{\infty} \frac{a_{P}^{i}(n)}{r^{n}} .
$$

Since the number on the left is transcendental, at least one of the numbers on the right is transcendental. This proves Theorem 10.

Corollary. If $r=2$ or 3 in Theorem 10 , then $\sum_{n=0}^{\infty} a_{P}(n) / r^{n}$ is transcendental.

Our last result deals with sequences of the kind considered in Theorem 2.

THEOREM 11. If $a_{s}$ is defined by (24), then

$$
\sum_{n=0}^{\infty} \frac{a_{s}(n)}{r^{n}}
$$

is transcendental if and only if $a_{s}$ does not have the form $a_{s}=\zeta^{\varepsilon_{1} \sigma}$, where $\varepsilon_{1} \not \equiv 0(\bmod r), r /\left(r, \varepsilon_{1}\right) \mid(k-1)$, and $\sigma$ is the sequence defined in Theorem 2. Otherwise (41) represents a number in $\mathbb{Q}(\zeta)$.

Proof. This is a consequence of $[10$, Theorem 1]. To see this, let

$$
f(z)=\sum_{n=0}^{\infty} a_{s}(n) z^{n}
$$

Since $M\left(a_{s}\right)=1$, it is clear that $a_{s}(n k+j)=a_{s}(n) a_{s}(j)$, for $0 \leq j \leq k-1$, by Lemma 7 . Thus

$$
f(z)=\sum_{j=0}^{k-1} \sum_{n=0}^{\infty} a_{s}(n k+j) z^{n k+j}=\sum_{j=0}^{k-1} a_{s}(j) z^{j} \sum_{n=0}^{\infty} a_{s}(n)\left(z^{k}\right)^{n}=p(z) f\left(z^{k}\right),
$$

where $p(z)$ is a polynomial of degree $k-1$. Since the constant term of $p(z)$ is $a_{s}(0)=1, p(0) \neq 0$. Further, the leading term of $p(z)$ is $a_{s}(k-1)$, so $p(z)$ has no rational roots other than \pm 1 , since its constant and leading terms are roots of unity and therefore units in the ring $\mathbb{Z}[\zeta]$. This shows that any positive rational number $\alpha \leq 1 / 2$ is admissible in the sense of [10] (taking $T z=z^{k}$ and $A(z)=p(z)$ in [10]). By Theorem 1 of [10], $f(1 / r)$ is transcendental if and only if $f(z)$ is a transcendental function. Since the coefficients of $f(z)$ are from a finite set, Szegö's theorem (see Bieberbach, Lehrbuch der Funktionentheorie, vol. 2, page 315) implies that $f(z)$ is transcendental if and only if the coefficient sequence $\left\{a_{s}(n)\right\}$ is not ultimately periodic. Theorem 11 now follows from Theorem 2 . 


\section{References}

[1] J. P. Allouche, 'Somme de chiffres et transcendance', Bull. Soc. Math. France 110 (1982), 279-295.

[2] J. P. Allouche, 'Automates finis en théorie des nombres', Expo. Math. 5 (1987), 239-266.

[3] J. P. Allouche and J. O. Shallit, 'Infinite products associated with counting blocks in binary strings', $J$. London Math. Soc., to appear.

[4] J. P. Allouche and J. Shallit, 'The ring of $k$-regular sequences', preprint.

[5] D. W. Boyd, J. Cook and P. Morton, On sequences of \pm 1 's defined by binary patterns, Dissertationes Mathematicae 283, Polish Scientific Publishers, Warszawa, 1989.

[6] G. Christol, T. Kamae, M. Mendès France and G. Rauzy, 'Suites algébriques, automates et substitutions', Bull. Soc. Math. France 108 (1980), 401-419.

[7] A. Cobham, 'Uniform tag sequences', Math. Systems Theory 6 (1972), 164-192.

[8] M. Dekking, M. Mendès France and A. van der Poorten, 'Folds! I, II, III', Math. Intelligencer 4 (1982), 130-138, 173-181, 190-195.

[9] H. Delange, 'Sur la fonction sommatoire de la fonction <<somme des chiffres $>>$ ', Enseign. Math. (2) 21 (1975), 31-47.

[10] J. H. Loxton and A. van der Poorten, 'Arithmetic properties of the solutions of a class of functional equations', J. Reine Angew. Math. 330 (1982), 159-172.

[11] J. H. Loxton and A. J. van der Poorten, 'Arithmetic properties of automata: regular sequences', J. Reine Angew. Math. 392 (1988), 57-69.

[12] M. Mendès France, 'Nombres algébriques et théorie des automates', Enseign. Math. (2) 26 (1980), 193-199.

[13] P. Morton and W. J. Mourant, 'Paper folding, digit patterns and groups of arithmetic fractals', Proc. London Math. Soc. (3) 59 (1989), 253-293.

[14] P. Morton, 'Connections between binary patterns and paperfolding', Séminaire de Théorie des Nombres, Bordeaux 2 (1990), 1-12.

Wellesley College

Wellesley, Massachusetts 02181

U.S.A.
37 William J. Heights

Framingham, Massachusetts 01701

U.S.A. 